\section{Absorption, Transmission And Reflection Of Light By The Specimen}

The following article is excerpted from the new RMS Handbook Contrast Techniques in Light Microscopy by S. Bradbury and P.J. Evennett. This book is available from Microscopy Today at a price of $\$ 34$ plus $\$ 5 \mathrm{~S} \& \mathrm{H}$.

These are perhaps the most fundamental interactions of light with matter, since they must often precede some of the other interactions described later. A good account of most of these will be found in the book by Slayter (1970). When light falls upon an object some of it will be reflected, some of it absorbed, and the remainder will be transmitted, phenomena which are familiar from everyday life. Most of the objects we observe are seen by the light that they reflect towards the eye, and their different surface appearances depend on the nature of this reflection. Some objects are transparent, enabling us to observe them by virtue of the light that they transmit - wine in a glass, church windows and colour transparencies are obvious examples. In all these cases, the nature of the light reflected or transmitted depends on the absorption of light by the object, and may be modified, for example by painting (reflection), or by incorporating a coloured component or dye (transmission).

After a component has been reflected, light will enter an object and may be partially transmitted (passed through the specimen) provided the specimen is not too thick. Light which is absorbed may be degraded to heat, initiate some form of photochemical reaction, or be re-radiated as phosphorescence or fluorescence (see Chapter 8). In transmitted-light microscopy, when working with relatively thin specimens, either naturally coloured or those which have been stained, absorption is the principal interaction which provides the contrast. Such objects, commonly called amplitude objects, affect the brightness of the light passing through them (see Figure 2.1). As far as the production of contrast is concerned, absorption is often the most important interaction which an object may have with the transmitted light. In some cases this amplitude reduction affects all the wavelengths so that the net result is a reduction in the brightness of the object. If, however, only certain wavelengths of the visible spectrum are absorbed (as when an object is stained with a coloured dye), there will be selective absorption of light, resulting in a change in colour of the transmitted light (see Figure 2.2). This provides the rationale for staining biological sections and for histochemical reactions which yield coloured endproducts. Other objects (e.g., some minerals, exoskeletons of insects) are themselves naturally coloured and again selective absorption provides the major interaction producing contrast.

When light strikes a surface, some or almost all of the radiation may be returned towards the source. If the surface is smooth (i.e., the irregularities are small compared with the wavelength of the radiation), as in a mirror, then we speak of specular reflection (see Figure 2.3a). This is characterized by the fact that the angle at which the light strikes the mirror (called the angle of incidence, $i$ in the figure) is equal to the angle at which it is reflected (the angle of reflection, $r$ in the figure). If the reflecting surface is rough, then the light is returned through a whole range of different angles

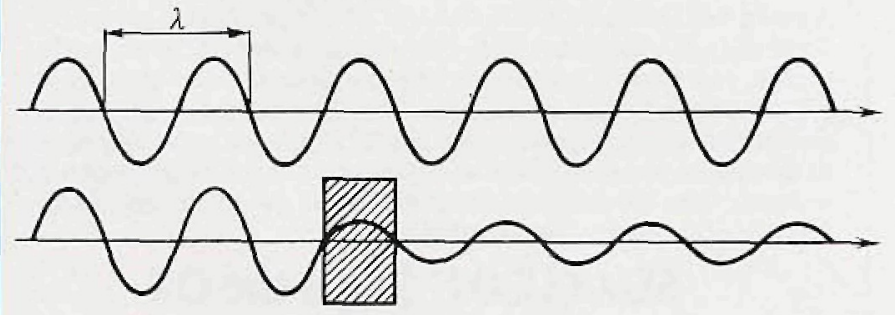

Figure 2.1: The conventional illustration (as sine waves) of two light beams travelling in the plane of the paper from left to right. $\lambda$ represents the wavelength, and the height of any point on the curve above or below the datum is the amplitude at that point. The lower beam passes through an amplitude object (shown cross-hatched) which does not affect the wavelength of the light but reduces the amplitude of the transmitted beam. and we speak of diffuse reflection (see Figure 2.3b). Reflection forms the basis of epi-illumination in microscopy.

The ratio of the intensities of reflected radiation to incident radiation is important, as it has a prominent role in affecting the contrast of the image formed by reflected light. The ratio is often called the reflectivity of the surface. It is related to the angle of incidence, the degree or otherwise of polarization of the light and the properties of the material at the surface onto which the radiation falls. There may also be variation according to the wavelength of the incident light. If the material is opaque, reflectivity is often high; with metals over $90 \%$ of the incident light may be reflected. Transparent materials, such as glass, many crystals and water, transmit the bulk of the incident radiation and reflectivity is therefore low. The reflectivity for an air/water interface and an air/glass interface would be approximately $0.02(2 \%)$ and $0.04(4 \%)$, respectively. Although this low reflectivity of air/glass is not of great value in microscopical examination of such surfaces, it is nevertheless important when considering the operation of the microscope lenses themselves, which contain many air/glass interfaces. In complex lens systems, even such a low reflectivity, occurring many times, can seriously degrade the contrast of the image produced. Accordingly, steps are now taken to reduce such unwanted reflection even further. This may be achieved by vacuum evaporating a layer, or layers, of some substance, such as maguesium fluoride, onto the glass surface. The thickness of such layers must be carefully controlled. By such means the reflectivity at coated surfaces may be reduced to a value of less than 0.005 . An alternative method for reducing unwanted reflectivity in an epi-illumination microscope system used with objectives of low numerical aperture is that used by firms such as Zeiss (termed by them the 'Antiflex' system). Linearly-polarized light is directed on to the specimen by a beam-splitter and passes through a quarter-wave plate mounted at the front lens of the objective. An analyser orientated at $90^{\circ}$ to the polarizer is fitted above the object beam-splitter. Unwanted reflections from the lens surfaces

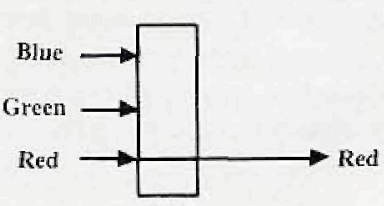

(a)

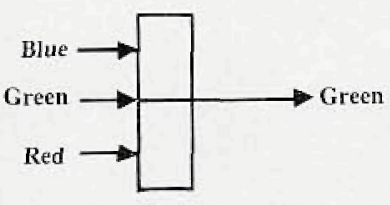

(b)
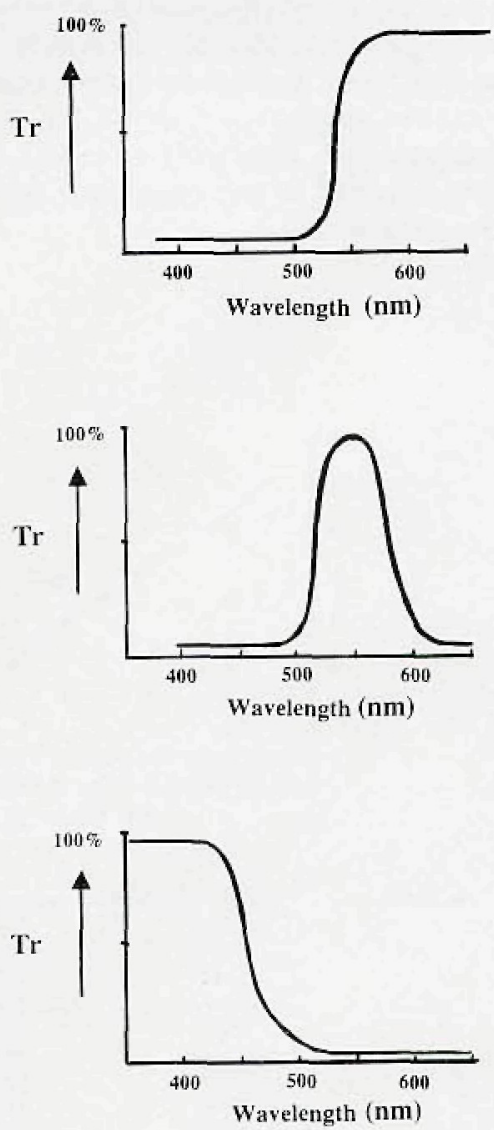

Figure 2.2. Varying effects of diflerent amplitude objects. Some amplitude objects affect only certain wavelengths of the light passing through them. Object (a) absorbs the shorter wavelengths, transmitting significantly only wavelengths above c. $550 \mathrm{~nm}$ : it appears RED. Object (b) absorbs wavelengths both below c. $500 \mathrm{~nm}$ and above c. $800 \mathrm{~nm}$ and appears GREEN, whilst object (c) absorbs strongly all wavelengths above c. $450 \mathrm{~nm}$ and thus 
pass upwards towards the eyepieces and are blocked from the image by the analyser. Rays reflected from the specimen, however, will have passed through the quarter-wave plate twice so that their polarization plane will be rotated through $90^{\circ}$ and will thus pass unimpeded through the analyser to form the image. A modification called reflection contrast microscopy, introduced by Ploem in 1975, allowed the extension of the basic approach used in the Antiflex objectives to oil immersion objectives of high aperture by various modifications, including fitting a central stop in the illuminating system. A good review of this technique is to be found in Ploem, 1995

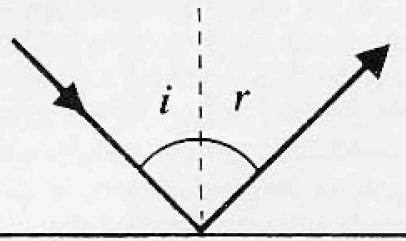

(a)

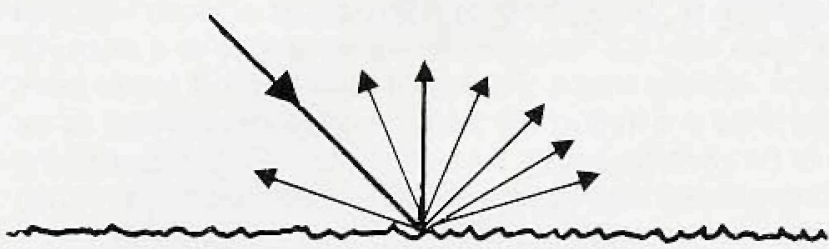

(b)

Figure 2.3: Specular and diffuse reflection. The upper diagram (a) illustrates specular reflection from a smooth surface. The angle of incidence (i) of the light is equal to the angle of reflection $(\mathrm{)})$. In diffuse reflection (b) the light from the incident beam is returned from the surface through a whole range of different angles.

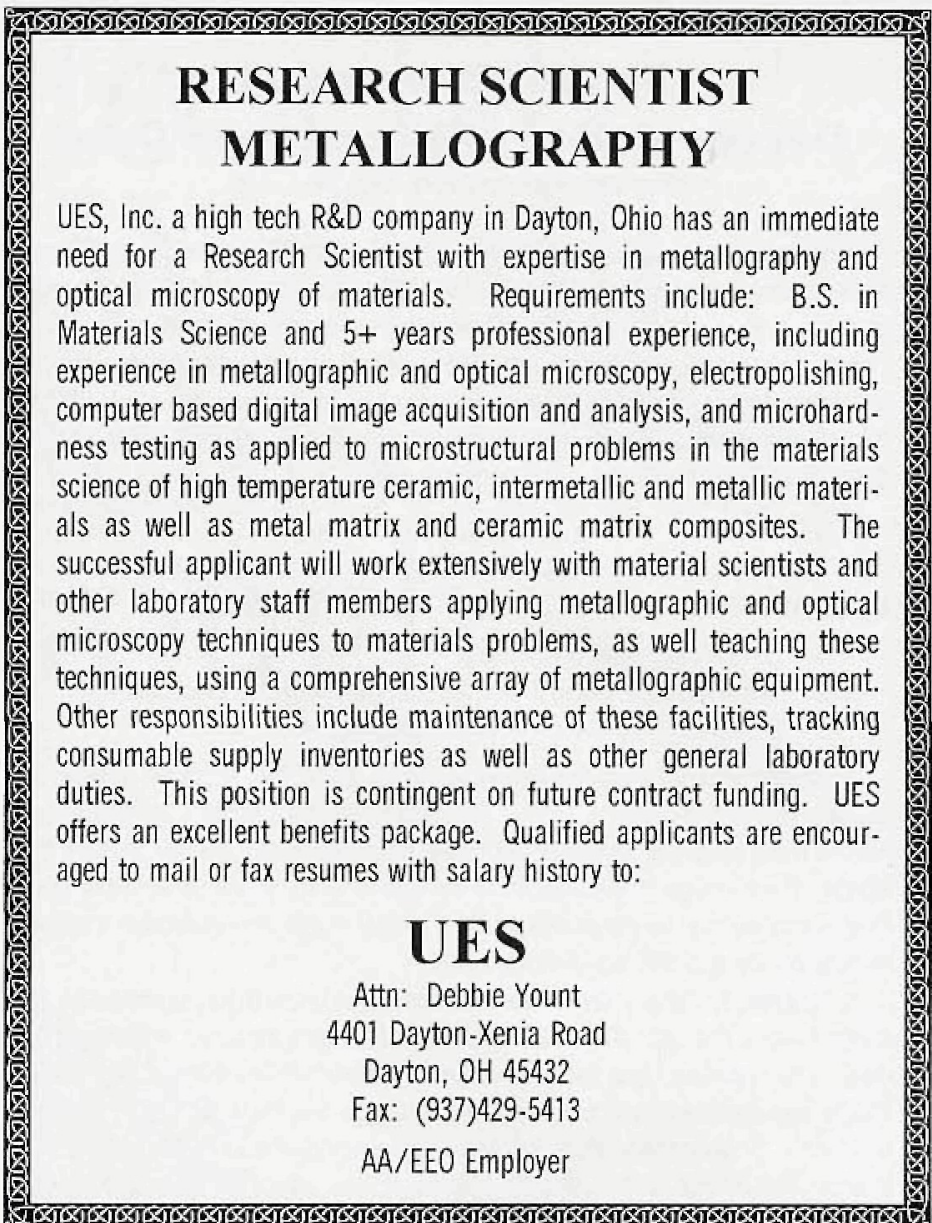

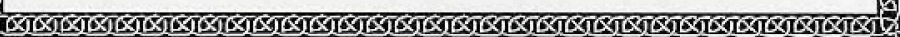

The Series 5 Robinson Detector

Whether you use conventional, field-emission or environmental SEMs or defect review tools, the Series 5 Robinson BSE Detector delivers unprecedented performance. More signal, less noise, and outstanding resolution. Call us to find out more.

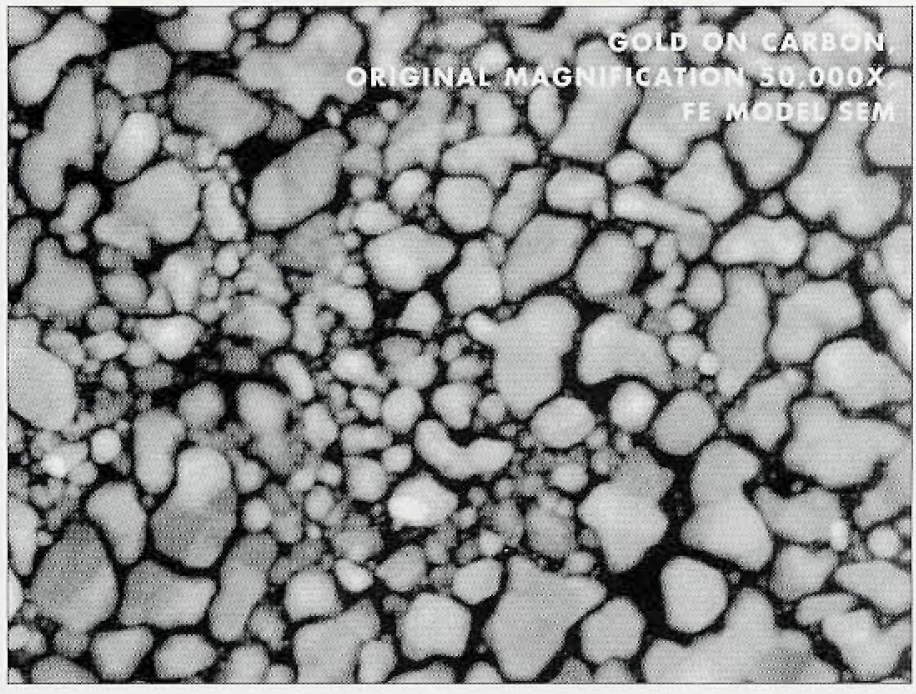

When image is everything, only the best will do. 